\title{
Ku70 is Associated with the Frequency of Chromosome Translocation in Human Lymphocytes After Radiation and T-Cell Acute Lymphoblastic Leukemia
}

\section{Zhenbo Cheng}

Central South University Third Xiangya Hospital https://orcid.org/0000-0001-6674-2697

\section{Yupeng Wang}

Hunan Provincial People's Hospital

\section{Lihuang Guo}

Central South University Third Xiangya Hospital

\section{Jiancheng Li}

Central South University Third Xiangya Hospital

\section{Wei Zhang}

Central South University Third Xiangya Hospital

\section{Conghui Zhang}

Central South University Third Xiangya Hospital

Yangxu Liu

Central South University Third Xiangya Hospital

\section{Yue Huang}

Central South University Third Xiangya Hospital

Keqian Xu ( $\nabla$ xukeqian@csu.edu.cn )

Central South University, Changsha

\section{Research}

Keywords: Ku70, chromosome translocation, human lymphocytes, radiation, T-cell acute lymphoblastic leukemia, radiation damage biomarker, therapy target

Posted Date: September 3rd, 2021

DOl: https://doi.org/10.21203/rs.3.rs-858112/v1

License: (c) (1) This work is licensed under a Creative Commons Attribution 4.0 International License. Read Full License 


\section{Abstract}

Background: Chromosome translocation is one of the most common chromosomal causes to T-cell acute lymphoblastic leukemia (T-ALL). Ku70 is one of the key factors of error-prone DNA repair that may end in translocation. So far, the direct correlation between Ku70 and translocation has not been assessed. Our study aimed to investigate the association of Ku70 and translocation in human lymphocytes after radiation and T-ALL.

Methods: Peripheral blood lymphocytes (PBLs) from volunteers and human lymphocyte cell line AHH1 were irradiated with X-rays to form chromosome translocations. The frequency of translocation was detected by fluorescence in situ hybridization (FISH), meanwhile, the expression of Ku70 was also detected by reverse transcription-quantitative polymerase chain reaction (RT-qPCR) and western blot. Furthermore, Ku70 interference, overexpression and chemical inhibition were used in AHH-1 cell lines to confirm the correlation. Finally, we detected the expression of Ku70 in T-ALL samples with or without translocation.

Results: Expression of Ku70 and frequencies of translocation were both significantly increased in PBLs after irradiated by X-rays, and a positive correlation between the expression (both mRNA and protein level) of Ku70 and the frequency of translocation was detected $(r=0.4877, P=0.004 ; r=0.3038, P=$ 0.0358 respectively). Moreover, Ku70 interference decreased the frequency of translocations while the frequency of translocations was not significantly affected after Ku70 overexpression. The expression of Ku70 and frequencies of translocation were both significantly increased in cells after irradiated combined with chemical inhibition $(P<0.01)$. The protein level and mRNA level of Ku70 in T-ALL with translocation were significantly higher than T-ALL with normal karyotype $(P=0.009, P=0.049$ respectively).

Conclusions: Ku70 is closely associated with the frequency of chromosome translocation in human lymphocytes after radiation and T-ALL. Ku70 might be a radiation damage biomarker and a potential tumor therapy target.

\section{Background}

Chromosome translocations are estimated to drive 20\% of cancer cases, especially hematopoietic malignancies such as T-cell acute lymphoblastic leukemia (T-ALL) [1]. Translocation has also been reported plays crucial roles in both the onset and progression of tumorigenesis $[2,3]$. The molecular mechanisms remain unclear.

DNA double-strand breaks (DSBs) are the initial events of chromosome translocation [4, 5]. Inappropriate end-joining events caused by error-prone DSBs repair, can lead to the formation of translocation, resulting in genomic instability and cell death [6], even oncogenic transformation [7].

Ku70, encoded by DNA repair gene $X R C C 6$, is one of the key factors of DSBs repair. During the DSBs repairing, the Ku heterodimer, Ku70 and Ku80, binds to the broken DNA as the first component of non- 
homologous end joining (NHEJ) [8]. Alternative end joining (Alt-EJ) is another important pathway to repair DSBs with high error propensity [9], and the PARP1 is a key factor to Alt-EJ [10]. Previous study has demonstrated that Ku70 facilitates NHEJ pathway and inhibits alt-EJ pathway [11]. In addition, it was also proposed that NHEJ factors such as Ku70 suppress the mobility of the broken ends and thus inhibit chromosome translocation $[12,13]$. Mouse embryonic fibroblasts deficient in Ku70 also show high frequency in translocations [14].

However, whether Ku70 is directly related to the frequency of translocation in human lymphocyte cells is largely unknown. In the present study, we investigated the potential association of Ku70 and translocation by combining the human primary lymphocyte cells and lymphocyte cell line after radiation for the first time, and finally verified in T-ALL samples. It was expected to uncover the underlying association of Ku70 and translocation in human lymphocyte cells after radiation, and provide basis for mechanism of translocation and tumor radiotherapy.

\section{Materials And Methods}

\section{Subjects}

The study involved 38 clinic samples (6 initial T-ALL with translocation, 19 initial T-ALL with normal karyotype and 13 non-tumor samples were obtained from remission bone marrow aspirates with less than $1 \%$ leukemic cells as normal controls) from Xiangya Hospital of Central South University (Changsha, China) from June 2018 to May 2021. Meanwhile, another 12 age matched healthy volunteers who had physical examinations at the same hospital were recruited. All of the volunteers had no X-ray radiation, chemical poisons touch, smoking history and karyotype abnormalities. All participants signed informed consent forms before participating in the study, and the study was approved by the Ethics Committee of the Third Xiangya Hospital of Central South University. The accession number for this approval was Quick19159. The details of all patients are presented in Table S1. Overall, the gender, age, ethnicity, and occupations were similar between T-ALL and remission patients. No statistically significant differences were identified (date not shown).

\section{Cells culture and treatments}

Peripheral blood was collected from each healthy volunteer and treated with X-rays. $0.5 \mathrm{ml}$ whole blood from each group was added into lymphocytes culture medium (Le Chen, Shanghai, China) at a humidified atmosphere of $37^{\circ} \mathrm{C}$ and $5 \% \mathrm{CO} 2$ in air for $72 \mathrm{~h}$. And the peripheral blood lymphocytes (PBLs) separated from the rest whole blood, were suspended in lymphocytes culture medium for further culturing. The lymphocyte cell lines AHH-1 were bought from ATCC (American Type Culture Collection, CRL-8146). The cell lines were cultured in RPMI 1640 (Thermo Fisher Scientific, USA) supplemented with 10\% FBS (Thermo Fisher Scientific, USA). The cultures were incubated at $37^{\circ} \mathrm{C}$ in a humidified atmosphere containing $5 \% \mathrm{CO} 2$.

\section{Bone marrow Karyotype}


A standard $72 \mathrm{~h}$ lymphocyte culture of bone marrow $(1-2 \mathrm{ml})$ from each patient performed to produce Metaphases for karyotyping. $\mathrm{G}$ banding was performed by a trypsin pretreatment of chromosomes followed by Giemsa staining. Chromosomes' analysis was done using MetaSystems Ikaros (ZEISS, Germany) and karyotypes were reported according to International System for Human Cytogenetic Nomenclature [15]. Karyotype analysis was performed using at least 20 Metaphases for each sample. Number was expanded to 100 metaphases in case of suspected mosaicism.

\section{RNA extraction, CDNA preparation and reverse transcription-quantitative polymerase chain reaction (RT- qPCR)}

Cellular RNAs were extracted using TRIZOL reagent (Takara Bio, Japan), according to the manufacturer's instructions. The RNA quality was assessed using a Nanodrop One (Thermo Fisher Scientific, USA) and agarose gel electrophoresis. cDNA was generated from $2 \mu \mathrm{g}$ of total RNA using M-MLV reverse transcriptase (Invitrogen, USA) with random primers. Quantitative PCR (qPCR) was performed on triplicate samples in a reaction mix of SYBR-Green (Takara Bio, Japan) using the ABI 7500 Real-Time PCR System (Applied Biosystems, USA). The conditions of PCR denaturation, annealing and extension were respectively $94^{\circ} \mathrm{C} 30 \mathrm{sec}, 37^{\circ} \mathrm{C} 30 \mathrm{sec}$, and $72^{\circ} \mathrm{C} 45 \mathrm{sec}$. Relative expression of Ku70 was normalized to GAPDH, and using the $2^{-\triangle \Delta C q}$ method [16]. The primers were synthesized by Sangon Biotech Co. Ltd. (Shanghai, China). Each RT-qPCR reaction was performed in triplicate. The primer sequences were as follows: Ku70 forward, 5'-GGTTTCAAGCCGTTGGTACTGC-3' and reverse, 5'-

CTCCAGACACTTGATGAGCAGAG-3'; GAPDH forward, 5'-ACCACAGTCCATGCCATCAC-3' and reverse, 5'TCCACCACCCTGTTGCTGTA-3'.

\section{Western blot analysis}

Total protein from cells was acquired using lysis buffer (KeyGen Biotech, Nanjing, China). Pierce BCA Protein Assay Kit (Thermo Fisher Scientific, USA) was used to calculate the protein concentration, 30 $\mathrm{\mu g}$ of protein was loaded on an $8 \%$ SDS-PAGE gel at $80 \mathrm{~V}$ for $1.5 \mathrm{~h}$ and transferred to $0.45 \mu \mathrm{m}$ PVDF membranes (Millipore, USA). The membranes, blocked with TBS- $0.05 \%$ Tween 20 (TBST) containing 5\% skimmed milk for $2 \mathrm{~h}$ at room temperature, were incubated with primary rabbit antibodies (Cell Signaling Technology, all diluted 1:1,000) overnight at $4^{\circ} \mathrm{C}$ and secondary HRP-conjugated goat anti-rabbit antibody (Cell Signaling Technology, 1:5000 diluted) for $1 \mathrm{~h}$ at room temperature in sequence. The protein blots were then visualized using an ECL kit (Beyotime Institute of Biotechnology, China). Semi-quantitative analysis of proteins was performed with Image Lab (Bio-Rad, USA). GAPDH was used as the loading control. Each western blot analysis experiment was repeated three times.

\section{Ku70 interference and overexpression}

Transfection was performed using Lipofectamine ${ }^{\circledR} 3000$ transfection reagent (Thermo Fisher Scientific, USA) according to the manufacturer's instructions. AHH-1 cells were transfected with shRNA of Ku70 (Genebio, Shanghai, China), targeted with sequence AACCAAGACCCGGACCTTTAA (Ku70-shRNA) or scrambled shRNA (negative control) targeted with TTCTCCGAACGTGTCACGT following the 
manufacturer's instructions to reduce the intracellular Ku70 levels, pcDNA3.1 or pcDNA3.1+Ku70 (Genebio, Shanghai, China) were transfected into cells to overexpress Ku70. Transfection effciency was assessed by western blotting after $72 \mathrm{~h}$ culturing. Cells were harvested and suspended in fresh lymphocytes culture medium, then irradiated by different dose $\mathrm{X}$-rays, followed another $72 \mathrm{~h}$ culturing, the expression of Ku70, PARP1 and the frequency of chromosome translocations in the cells were respectively explored by western blot and fluorescence in situ hybridization.

\section{Chemical inhibition}

PARP1 inhibitor Olaparib (Abmole, USA) at $5 \mu \mathrm{M}$ or $0.1 \%$ DMSO (solvent control) was added into the cells culture medium $3 \mathrm{~h}$ before X-rays. After $72 \mathrm{~h}$ culturing, the expression of Ku70, PARP1 and the frequency of chromosome translocations in cells were respectively explored by western blot and fluorescence in situ hybridization.

\section{Fluorescence in situ hybridization (FISH)}

FISH was used to detect the chromosome translocations in human PBLs and cell lines after X-rays as previously described [17]. Metaphases were harvested after co-cultured with colchicine for $2 \mathrm{~h}$. Chromosomes 1 and 4 were painted green by in situ hybridization with composite probes labelled with SYBR green (Cytocell, UK), chromosomes 2 were painted red by in situ hybridization with composite probes labelled with Rhodamine B (Cytocell, UK). The observed frequency of translocations $\left(F_{\mathrm{p}}\right)$ detected by FISH represents the frequency between painted chromosomes 1, 2 and 4 and the remaining counterstained chromosomes. To compare $F_{\mathrm{p}}$ with the values for translocations detected by the conventional method that detects aberrations involving the entire chromosome set, it is necessary to estimate the genome-equivalent frequency of translocations $\left(F_{\mathrm{G}}\right)$. Thus, since the fraction of the total genomic DNA content represented by painted chromosomes 1, 2 and 4 to the total genome is 0.228 for males and 0.224 for females, $F_{\mathrm{p}}$ was multiplied by 2.771 for males and 2.806 for females to estimate $F_{G}$; the basic method used is essentially that described by Pearce et al [18]. 400 metaphase splitting images were observed for each sample by three observers. The experiments were repeated three times.

\section{Comet assay (neutral condition)}

The relative amount of DNA double-strand breaks (DSBs) was detected by neutral comet assay in PBLs as previously described [19]. Slides prepared were evaluated using a fluorescence microscope and the CASP software (CASP, Wroclaw, Poland). Data was expressed as tail intensity (\% Tail DNA). The experiments were repeated three times.

\section{Statistical analysis}

Analysis was performed by GraphPad Prism 7.0 (GraphPad Software, San Diego, CA). Measurement data was expressed as the mean \pm standard deviation (SD). Two group comparisons were analyzed by a two- 
tailed Student's t-test. One-way ANOVA and two-way ANOVA were used for multiple comparisons. $P<0.05$ was considered to be statistically significant.

\section{Results}

\section{There was a dose- and time-response of radiation on Ku70 in human PBLs}

We explored the expression of Ku70 in human peripheral blood lymphocytes (PBLs) after X-rays first. Figure 1A showed that the expression of Ku70 mRNA in PBLs accumulated in 1Gy and 2Gy X-ray $(P<0.05)$. Besides, Ku70 also showed the increment in protein level after $1 \mathrm{~Gy}$ and $2 \mathrm{~Gy} \mathrm{X}$-ray (Figure.1B and $1 \mathrm{C}, P<0.05$ ). Radiation exposure in 0.5Gy did not change the expression of Ku70 in human PBLs (Figure.1A, 1B and 1C, $P>0.05$ ). Neutral comet assay showed after exposure to $0.5 \mathrm{~Gy}, 1 \mathrm{~Gy}$ and 2Gy radiation, the DNA damage extent markedly increased in PBLs immediately $(0 \mathrm{~h})$, and only $1 \mathrm{~Gy}$ and 2Gy group still showed markedly increased at $72 \mathrm{~h}$ (Table $1, P<0.01, P<0.001$ respectively). The PBLs DNA damage at $72 \mathrm{~h}$ reduced markedly in all groups when compared with that at $0 \mathrm{~h}$ (Figure.1D and 1E, $P<0.001$ ).

\section{Frequency of translocations showed a positive correlation with levels of Ku70}

To reveal whether Ku70 is related to the frequency of translocation in human primary lymphocyte cells, PBLs were irradiated with increasing dose of X-rays and left to repair for $72 \mathrm{~h}$. Chromosome translocations were observed by FISH (Figure. 2A). The whole genome translocation frequencies in different groups were shown in Figure. 2B, the frequency of translocations detected after 0.5Gy, 1Gy and 2Gy X-rays were significantly higher than that in non-irradiated control $(P<0.05, P<0.01$ and $P<0.001$ respectively). Furthermore, Pearson correlation analysis was performed to explore the relevance of Ku70 and chromosome translocations in human PBLs after X-rays. The frequency of chromosome translocations showed a moderate positive correlation with the level of Ku70 mRNA (Figure. 2C, $r=$ $0.4877, P=0.0004$ ) and a weak positive correlation with the level of Ku70 protein (Figure. 2D, $r=0.3038$, $P=0.0358)$.

\section{Ku70 interference decreased the frequency of chromosome translocations}

To further prove the correlation between Ku70 and chromosomal translocation, western blot was performed to confirm the interference or overexpression efficiency and the effect on PARP1 expression. Ku70 was significantly reduced accompanied by a significant increase in PARP1 (Figure.3A and 3B, $P<$ 0.01 and $P<0.05$ respectively), while PARP1 level changed rarely when Ku70 was overexpressed (Figure.3D and 3E, Ku70 $P<0.01$ and PARP1 $P>0.05$ ). To investigate the role of Ku70 in response to chromosome translocations, the Ku70 interference or overexpression cells were treated with $0.5 \mathrm{~Gy}, 1 \mathrm{~Gy}$ and 2Gy X-rays respectively. It was observed that the frequency of chromosome translocations was

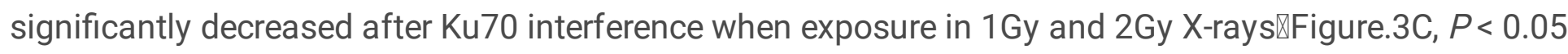
and $P<0.001$ respectively $\llbracket$. However, the frequency of translocations was not significantly affected by Ku70 overexpression $\otimes$ Figure.3F, $P>0.05 \rrbracket$. 


\section{PARP1 inhibitor increased expression of Ku70 and translocations after X-rays}

To further explore the effect of the PARP1 inhibitor on Ku70 and translocations, Olaparib was used. We first observed the connection of PARP1 with irradiation dose before inhibitor used. As we can see from Figure.4A and 4B, the PARP1 expression in cells increased with increasing X-rays dose. The expression of PARP1 decreased apparently in $3 \mathrm{~h}$ after pre-treated with Olaparib (Figure.4C and 4D, $P<0.01$ ), and Ku70 was not significantly affected (Figure.4C and $4 D, P>0.05$ ). Ku70 only increased in cells treated with Olaparib after 2Gy X-ray accompanied by slight increase of PARP1 (Figure. 4E and 4F, $P<0.01, P<0.05$ ). Compared to cells treated with 2Gy X-rays alone, only Ku70 in cells treated with Olaparib in combination with 2Gy X-rays increased significantly (Figure. 4G, $P<0.01$ ). The frequency of chromosome translocations also increased significantly in cells treated with Olaparib than cells treated with DMSO in combination with 1 Gy or 2 Gy X-rays (Figure. $6 \mathrm{H}, P<0.05$ and $P<0.01$ ).

\section{Ku70 in T-ALL was significant higher, especially in T-ALL with translocation}

Ku70 protein level in T-ALL (with translocation or normal karyotype) was significant higher than that in the normal control (Figure 5A and 5B, $P=0.002$ and $P=0.041$ respectively), and Ku70 protein level in translocation group was significantly higher than in normal karyotype group (Figure 5A and 5B, $P$ $=0.009$ ). The same phenomenon was found in the mRNA level of Ku70 (Figure 5C, $P=0.005, P=0.011$ and $P=0.049$ respectively).

\section{Discussion}

In the present study, we first found there was a dose-and time-response of radiation on DNA doublestrand breaks (DSBs) in human peripheral blood lymphocytes (PBLs) irradiated by X-rays, both of the expression of Ku70 and the frequency of chromosome translocations were increased with X-rays dose. A positive correlation between the expression of Ku70 and the frequency of chromosome translocation was observed in human PBLs irradiated by X-rays. The frequency of chromosome translocations significantly decreased in Ku70-deficient cells. PARP1 slightly increased in response to DSBs irradiated by X-rays. The expression of Ku70 and the frequency of chromosome translocations increased in lymphocyte cell lines treated with PARP1 inhibitor and X-rays. Finally, we verified our results in clinical samples, finding that the expression of Ku70 was significantly higher in T-cell acute lymphoblastic leukemia (T-ALL) patients than in remission patients, especially in T-ALL with translocation. These all findings provide convincing evidence that Ku70 is closely associated with the frequency of chromosome translocation in human lymphocytes after radiation and T-ALL.

Previous study has shown that the level of Ku70 is related to hepatocellular carcinoma prognostic factors, such as the tumor size, tumor nodule number, and distant metastasis [20]. And clinical pathological analysis shows that the high expression of Ku70 is associated with poor prognosis [21]. We speculated that there was a novel mechanism of Ku70-mediated genetic alterations (such as translocation) and hematological carcinogenesis. 
Radiotherapy induces cancer cells death mainly by causing DSBs, which lead to chromosome instability and translocation [22]. X-ray radiation is a major therapeutic modality for cancer treatment since it kills tumor cells by inducing DSBs. In recent years, many researchers have demonstrated the key role of Ku70 in DSBs repair, while few studies have focused on its role in the formation of chromosome translocation. In our study, significant up-regulation of Ku70 indicated the active role of Ku70 in X-rays irradiated PBLs and formation of chromosome translocation. This is consistent with Sheike et al. report that the level of Ku70 up-regulates in human peripheral blood mononuclear cells after $\mathrm{y}$-rays [23].

Neutral comet assay mainly used to assess the severity of DSBs [24], and DSBs are initial events for the formation of chromosome translocations $[4,5]$. Therefore, in our study neutral comet assay was used to detect the dose- and time-response of radiation on DSBs in PBLs to evaluate the formation of chromosome translocation. The result verified that it was difficult to repair more serious DSBs caused by high X-rays [25], and frequency of chromosome translocations increased with increasing X-rays dose within limits. To strengthen the comparison between radiation groups, the experiment was strictly controlled to ensure the minimal DNA damage in the control group ( $0 \mathrm{~Gy}$ ). Furthermore, increased DNA double strand breaks were observed after 0.5 Gy X-ray, considering the high sensibility for T cells to Xrays [26]. However, the repair of minimal DSBs might be accomplished rapidly, so the DSBs could not be detected in $0.5 \mathrm{~Gy}$ at $72 \mathrm{~h}$. These findings could also be used to evaluate the biological effects of various $\mathrm{X}$-rays radiation doses on human PBLs.

Increasing study indicated a pivotal role of chromosome translocation in the progression of tumorigenesis [27], and we speculated that Ku70 might also participate in the formation of chromosome translocation. In our study, the frequency of chromosome translocations increased with the doses of Xrays and showed a positive correlation with the expression of Ku70, consistent with Ghezraoui reported [28]. Previous researchers also explore that Ku protein regulates end-resection of unbroken forks and HRmediated replication restart [9], and has inhibitory effect on other repair pathways [29], for example, PARP1 is suppressed by Ku70 because of its high affinity to DSBs [30]. Besides, Ku protein also plays an important role in regulating DSB end-processing during HR and Alt-EJ [31]. Previous studies have also demonstrated that promotion of NHEJ results in chromosome aberrations and cell death [32]. Therefore, we concluded that high Ku70 expression might inhibit the other repair pathways and promote the errorprone repair pathway ending in translocations.

In order to further explore the mechanistic role of Ku70 in chromosome translocation, Ku70 expression was silenced by shRNA in AHH-1 cell lines followed by X-rays. It was observed that PARP1 slightly increased in response to DSBs, which was consistent with previous literature that X-ray irradiation produces dose-dependent increases in poly (ADP-ribose) (PAR) formation, an index of PARP1 activation [33]. Meanwhile, the frequency of chromosome translocations significantly decreased in Ku70-deficient cells. However, Ku70 overexpression did not increase the frequency of translocations. We speculate that, with sufficient Ku70, continued increase of Ku70 will not affect translocation. 
Olaparib is one of the commonly used PAPR1 inhibitors for antitumor in clinic. PARP1 inhibition could reduce alt-NHEJ frequency and the frequency of DSB-induced chromosome translocations in Ku-deficient cells $[34,35]$. To further explore whether NHEJ is the predominant pathway involve in X-ray induced translocation or not, Olaparib was used. Our results showed that the expression of Ku70 and the frequency of chromosome translocations increased when cells treated with Olaparib (PARP1 inhibitor) compared to cells treated with X-rays alone, indicating that NHEJ is also an important pathway involve in translocation. Previous study has also demonstrated that Ku70/80 and PARP1 proteins compete for binding to DSBs for repair [36]. Interestingly, in the Fig. 4E, PARP1 was not suppressed by Olaparib in 2 Gy X-rays, it was found that high expression of Ku70 seems to reduce the effects of Olaparib.

Despite sufficient powerful mastery and analysis, one of the limitations of our study might be the relatively small clinic sample size (only 38 T-ALL collected in three years), which does not allow definite conclusions. Another limitation is only experiments in vitro performed. More researches especially large samples combined with experiments in vivo are needed in the future to focus on radiation therapy, finding and manufacturing inhibitors or regulating epigenetic modification of Ku70 to achieve the curative effect of tumors.

Nevertheless, this study has several strengths, including combination of using bone marrow, peripheral blood lymphocytes (PBLs) and lymphocyte cell lines for analysis, case-control and inclusion of patients with standard clinically defined T-ALL. High expression of Ku70 was found in T-ALL, especially in T-ALL with chromosome translocation, and a high frequency chromosome translocation model using PBLs was constructed by exposing in X-ray. Moreover, we also found that high expression of Ku70 may reduce the effects of PARP1 inhibition. This all could be useful in guiding future research into molecular mechanisms and therapy of T-ALL.

In conclusion, the present study suggested that Ku70 is closely associated with the frequency of chromosome translocation in human lymphocytes after radiation and T-ALL for the first time. Ku70 might be a radiation damage biomarker and a potential tumor therapy target.

\section{Declarations}

\section{Acknowledgements}

The authors are grateful to all the participants in this study.

\section{Authors' contributions}

Zhenbo Cheng and Yupeng Wang conceived and designed the study. Yue Huang and Lihuang Guo collected the data, managed the data base, analysed the data. Jiancheng Li and Wei Zhang contributed in the interpretation of the data. Conghui Zhang and Yangxu Liu performed the experiments. Zhenbo Cheng and Keqian Xu drafted and revised the manuscript. All authors have approved the final version of the manuscript to be published. 


\section{Funding}

This study was supported by grants from the National Natural Science Foundation of China (81471499 to KQ.X.), the Natural Science Foundation of Hunan province (2019JJ40347 to KQ.X.) and the Fundamental Research Funds for the Central Universities of Central South University (2018zzts243 to ZB.C.).

\section{Availability of data and materials}

The materials used and data sets analysed in the current study are available from the corresponding author on reasonable request.

\section{Ethics approval}

The study was approved by the Ethics Committee of the Third Xiangya Hospital of Central South University. The accession number for this approval was Quick19159.

\section{Consent to participate}

All participants signed informed consent forms before participating in the study.

\section{Consent for publication}

All participants agreed to publish.

\section{Competing interests}

The authors declare that they have no competing interests.

\section{Author details}

${ }^{1}$ Department of Laboratory Medicine, the Third Xiangya Hospital, Central South University, Changsha 410013, P.R. China; ${ }^{2}$ Department of Laboratory Medicine, Xiangya School of Medicine, Central South University, Changsha 410013, P.R. China;

${ }^{3}$ Department of Medical Laboratory, Hunan Provincial People's Hospital, The First Affiliated Hospital of Hunan Normal University, Changsha 410005, P.R. China

\section{References}

1. Mitelman F, Johansson B, Mertens F.The impact of translocations and gene fusions on cancer causation. Nat Rev Cancer. 2007; 7(4):233-45.

2. Byrne M, Wray J, Reinert B, Wu Y, Nickoloff J, et al.Mechanisms of oncogenic chromosomal translocations. Ann N Y Acad Sci. 2014; 1310:89-97. 
3. Roukos V, Misteli T.The biogenesis of chromosome translocations. Nat Cell Biol. 2014; 16(4):293300.

4. Cho MS, Lee JK, Bae KS, Han EA, Jang SJ, et al.Retrospective biodosimetry using translocation frequency in a stable cell of occupationally exposed to ionizing radiation. J Radiat Res. 2015; 56(4):709-16.

5. Agarwal S, Tafel AA, Kanaar R.DNA double-strand break repair and chromosome translocations. DNA Repair (Amst). 2006; 5(9-10):1075-81.

6. Jeggo PA, Geuting V, Löbrich M.The role of homologous recombination in radiation-induced doublestrand break repair. Radiother Oncol. 2011; 101(1):7-12.

7. Aparicio T, Baer R, Gautier J.DNA double-strand break repair pathway choice and cancer. DNA Repair (Amst). 2014; 19:169-75.

8. Chanut P, Britton S, Coates J, Jackson SP, Calsou P.Coordinated nuclease activities counteract Ku at single-ended DNA double-strand breaks. Nat Commun. 2016; 7:12889.

9. Teixeira-Silva A, Ait Saada A, Hardy J, Iraqui I, Nocente MC, et al.The end-joining factor Ku acts in the end-resection of double strand break-free arrested replication forks. Nat Commun. 2017; 8(1):1982.

10. Iliakis G, Murmann T, Soni A.Alternative end-joining repair pathways are the ultimate backup for abrogated classical non-homologous end-joining and homologous recombination repair: Implications for the formation of chromosome translocations. Mutat Res Genet Toxicol Environ Mutagen. 2015; 793:166-75.

11. Kai M.Roles of RNA-Binding Proteins in DNA Damage Response. Int J Mol Sci. 2016; 17(3):310.

12. Roukos V, Voss TC, Schmidt CK, Lee S, Wangsa D, et al.Spatial dynamics of chromosome translocations in living cells. Science. 2013; 341(6146):660-4.

13. Soutoglou E, Dorn JF, Sengupta K, Jasin M, Nussenzweig A, et al.Positional stability of single doublestrand breaks in mammalian cells. Nat Cell Biol. 2007; 9(6):675-82.

14. Ferguson DO, Sekiguchi JM, Chang S, Frank KM, Gao Y, et al.The nonhomologous end-joining pathway of DNA repair is required for genomic stability and the suppression of translocations. Proc Natl Acad Sci U S A. 2000; 97(12):6630-3.

15. Simons A, Shaffer LG, Hastings RJ.Cytogenetic Nomenclature: Changes in the ISCN 2013 Compared to the 2009 Edition. Cytogenet Genome Res. 2013; 141(1):1-6.

16. Livak KJ, Schmittgen TD.Analysis of relative gene expression data using real-time quantitative PCR and the 2(-Delta Delta C(T)) Method. Methods. 2001; 25(4):402-8.

17. Nakano M, Kodama $Y$, Ohtaki K, Itoh M, Delongchamp R, et al.Detection of stable chromosome aberrations by FISH in A-bomb survivors: comparison with previous solid Giemsa staining data on the same 230 individuals. Int J Radiat Biol. 2001; 77(9):971-7.

18. Pearce MS, Salotti JA, Little MP, McHugh K, Lee C, et al.Radiation exposure from CT scans in childhood and subsequent risk of leukaemia and brain tumours: a retrospective cohort study. Lancet. 2012; 380(9840):499-505. 
19. Garcia O, Romero I, González JE, Mandina T.Measurements of DNA damage on silver stained comets using free Internet software. Mutat Res. 2007; 627(2):186-90.

20. Hsu CM, Yang MD, Chang WS, Jeng LB, Lee MH, et al.The contribution of XRCC6/Ku70 to hepatocellular carcinoma in Taiwan. Anticancer Res. 2013; 33(2):529-35.

21. Zhang $T$, Zhang $X$, Shi $W, X u J$, Fan $H$, et al.The DNA damage repair protein Ku70 regulates tumor cell and hepatic carcinogenesis by interacting with FOXO4. Pathol Res Pract. 2016; 212(3):153-61.

22. Tian H, Gao Z, Li H, Zhang B, Wang G, et al.DNA damage response--a double-edged sword in cancer prevention and cancer therapy. Cancer Lett. 2015; 358(1):8-16.

23. Shelke S, Das B.Dose response and adaptive response of non-homologous end joining repair genes and proteins in resting human peripheral blood mononuclear cells exposed to $y$ radiation. Mutagenesis. 2015; 30(3):365-79.

24. Goutham HV, Mumbrekar KD, Vadhiraja BM, Fernandes DJ, Sharan K, et al.DNA double-strand break analysis by $\mathrm{Y}-\mathrm{H} 2 \mathrm{AX}$ foci: a useful method for determining the overreactors to radiation-induced acute reactions among head-and-neck cancer patients. Int J Radiat Oncol Biol Phys. 2012; 84(5):e607-12.

25. Zhang M, Cao G, Guo X, Gao Y, Li W, et al.A Comet Assay for DNA Damage and Repair After Exposure to Carbon-Ion Beams or X-rays in Saccharomyces Cerevisiae. Dose Response. 2018; 16(3):1559325818792467.

26. Hu Q, Xie Y, Ge Y, Nie X, Tao J, et al.Resting T cells are hypersensitive to DNA damage due to defective DNA repair pathway. Cell Death Dis. 2018; 9(6):662.

27. Li W, Bai X, Li J, Zhao Y, Liu J, et al.The nucleoskeleton protein IFFO1 immobilizes broken DNA and suppresses chromosome translocation during tumorigenesis. Nat Cell Biol. 2019; 21(10):1273-85.

28. Ghezraoui H, Piganeau M, Renouf B, Renaud JB, Sallmyr A, et al.Chromosomal translocations in human cells are generated by canonical nonhomologous end-joining. Mol Cell. 2014; 55(6):829-42.

29. Kim JS, Krasieva TB, Kurumizaka H, Chen DJ, Taylor AM, et al.Independent and sequential recruitment of NHEJ and HR factors to DNA damage sites in mammalian cells. J Cell Biol. 2005; 170(3):341-7.

30. Zhang Y, Jasin M.An essential role for CtIP in chromosomal translocation formation through an alternative end-joining pathway. Nat Struct Mol Biol. 2011; 18(1):80-4.

31. Mendez-Dorantes C, Bhargava R, Stark JM.Repeat-mediated deletions can be induced by a chromosomal break far from a repeat, but multiple pathways suppress such rearrangements. Genes Dev. 2018; 32(7-8):524-36.

32. Sunada S, Nakanishi A, Miki Y.Crosstalk of DNA double-strand break repair pathways in poly(ADPribose) polymerase inhibitor treatment of breast cancer susceptibility gene 1/2-mutated cancer. Cancer Sci. 2018; 109(4):893-99.

33. Sheng $C$, Chen $H$, Wang $B$, Wang $C$, Lin $L$, et al.Poly(ADP-ribose) polymerase activation mediates synchrotron radiation $\mathrm{X}$-ray-induced damage of rodent testes by exacerbating DNA damage and apoptotic changes. Int J Radiat Biol. 2014; 90(7):580-6. 
34. Frit P, Barboule N, Yuan Y, Gomez D, Calsou P.Alternative end-joining pathway(s): bricolage at DNA breaks. DNA Repair (Amst). 2014; 17:81-97.

35. Mansour WY, Borgmann K, Petersen C, Dikomey E, Dahm-Daphi J.The absence of Ku but not defects in classical non-homologous end-joining is required to trigger PARP1-dependent end-joining. DNA Repair (Amst). 2013; 12(12):1134-42.

36. Esposito MT, Zhao L, Fung TK, Rane JK, Wilson A, et al.Synthetic lethal targeting of oncogenic transcription factors in acute leukemia by PARP inhibitors. Nat Med. 2015; 21(12):1481-90.

\section{Tables}

Table 1 Human peripheral blood lymphocytes X-ray dose response $(n=3)$

Time after treated $\quad 0 \mathrm{~h} \quad 72 \mathrm{~h}$

with X-ray

\begin{tabular}{|c|c|c|c|c|c|c|c|c|}
\hline X-ray dose (Gy) & 0 & 0.5 & 1 & 2 & 0 & 0.5 & 1 & 2 \\
\hline$\%$ Tail DNA ${ }^{a}$ & 0.01 & $3.64^{\star}$ & 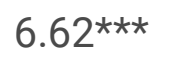 & 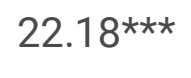 & 0.03 & 0.23 & $2.07^{\star \star}$ & $4.85^{\star \star \star}$ \\
\hline $\mathrm{SD}^{\mathrm{b}}(\%)$ & 0.54 & 7.80 & 1.00 & 11.87 & 1.62 & 1.30 & 5.03 & 2.33 \\
\hline
\end{tabular}

a\% Tail DNA: Mean percentage of total DNA, calculated from averages of three repeats.

bSD: standard deviation. One-way ANOVA was performed to compare each treated dose to negative controls $\left({ }^{\star} P<0.05,{ }^{\star \star} P<0.01,{ }^{* \star \star} P<0.001\right)$.

\section{Figures}




\section{C}
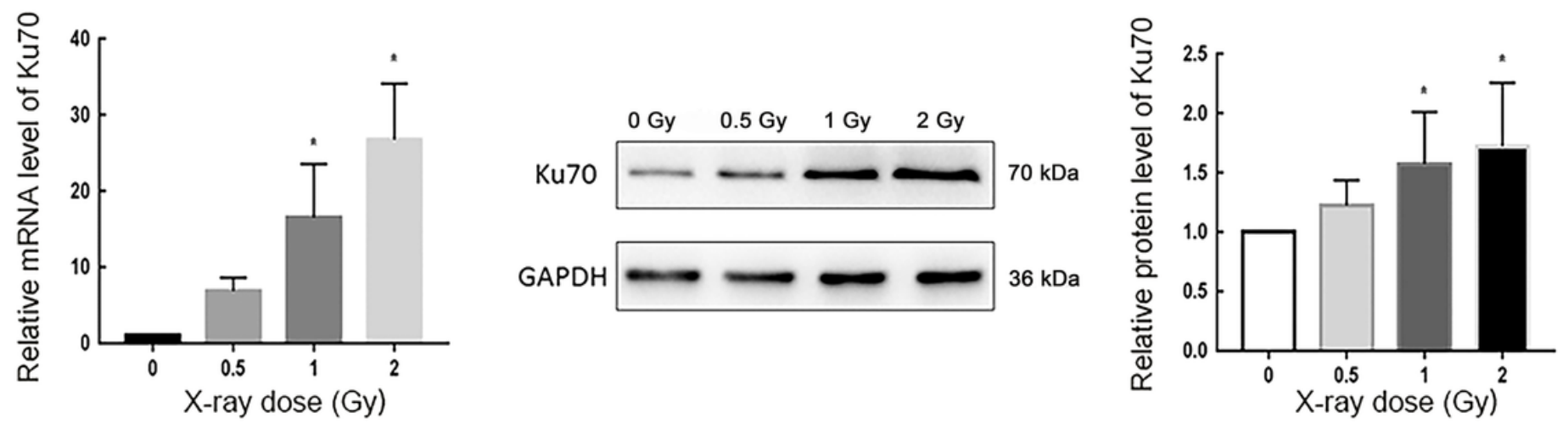

$D$

X-ray dose (Gy)

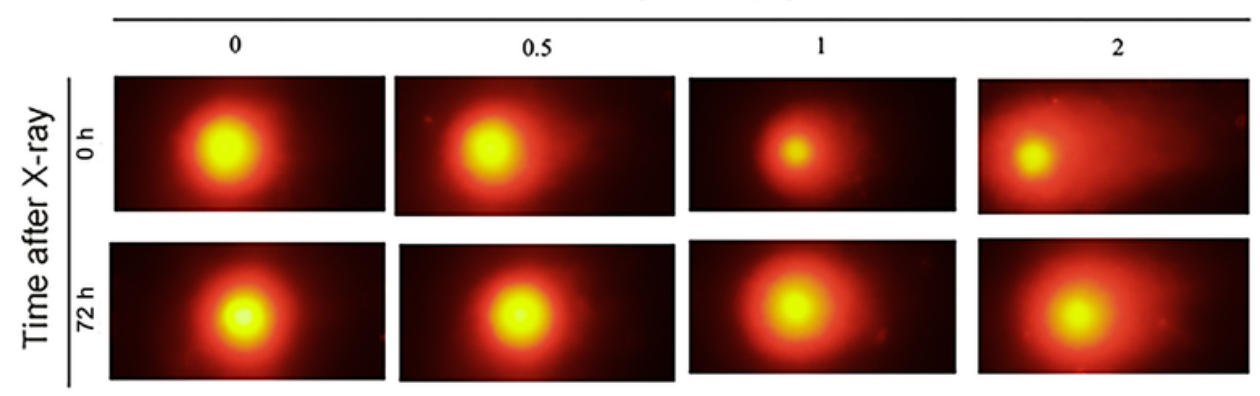

$E$

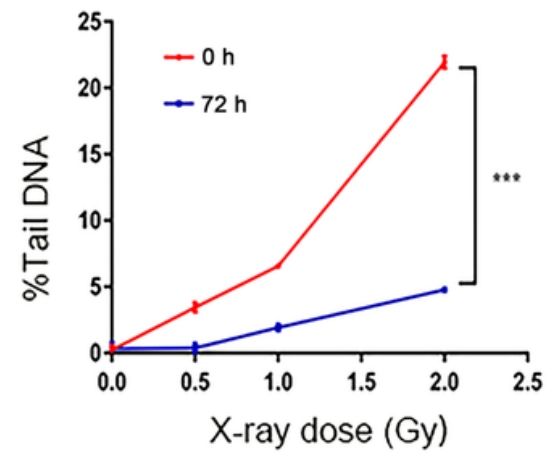

Figure 1

There was a dose- and time-response of radiation on DSBs and Ku70 in human PBLs. (A) RT-qPCR analysis of Ku70 mRNA. The expression of Ku70 mRNA accumulated in 1Gy and 2Gy X-irradiated PBLs.

(B) and (C) Representative western blot images and analysis. Ku70 showed the increment in protein level after $1 \mathrm{~Gy}$ and $2 \mathrm{~Gy}$ X-ray radiations. (D) Representative images of neutral comet assay. PBLs were isolated and subjected to $0.5 \mathrm{~Gy}, 1 \mathrm{~Gy}$ and $2 \mathrm{~Gy} \mathrm{X}$-ray radiations and then respectively subjected to the neutral comet assay after $0 \mathrm{~h}$ and $72 \mathrm{~h}$. (E) Result of Comet images analyzed. Data are presented as mean value \pm SD of three independent experiments. DSBs: DNA double-strand breaks; PBLs: Peripheral blood lymphocytes. ${ }^{*}$ indicated that $\mathrm{P}<0.05, * * *$ indicated that $\mathrm{P}<0.001$ compared to the control. 


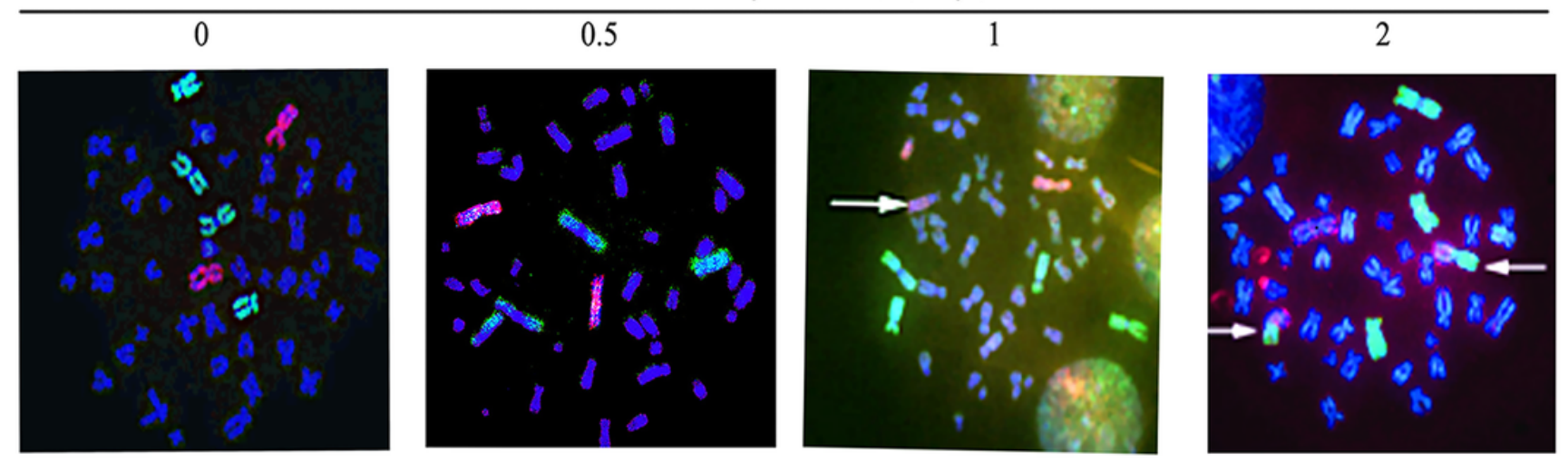

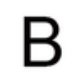

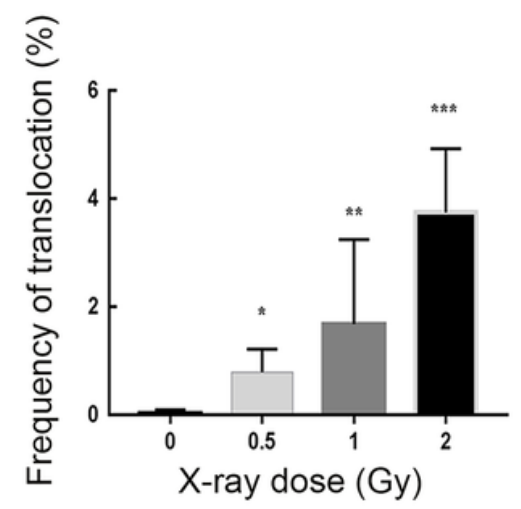

C

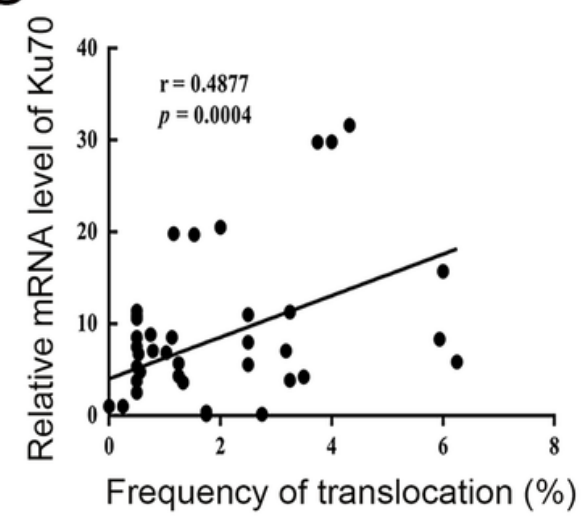

$\mathrm{D}$

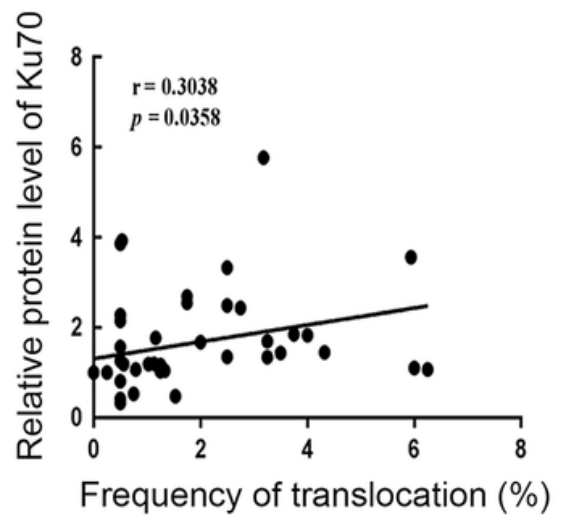

\section{Figure 2}

The frequency of chromosome translocations showed a positive correlation with the level of Ku70. (A) Representative images of photomicrographs FISH showing painted human chromosome 1, 4 (green) and 2 (red) in interphase lymphocytes after X-rays. Chromosomes translocations were showed with the arrows. (B) FISH analysis showing that X-ray radiations increased the frequency of chromosome translocations of PBLs. (C) Pearson correlation analysis of chromosome translocations and Ku70 mRNA showed a significant positive correlation of the frequency of chromosome translocations versus the expression of Ku70 mRNA (Pearson $r=0.4877 ; P=0.004$ ). The plots $(n=48)$ represent 12 volunteers $P B L s$ in 4 dose of X-rays (D) Pearson correlation analysis of chromosome translocations and Ku70 protein level showed a significant positive correlation of the frequency of chromosome translocations versus the expression of $\mathrm{Ku} 70$ protein (Pearson $r=0.3038 ; P=0.0358)$. The plots $(n=48)$ represent 12 volunteers PBLs in 4 dose of $X$-rays. PBLs: Peripheral blood lymphocytes. *indicated that $\mathrm{P}<0.05$, $\star *$ indicated that $\mathrm{P}$ $<0.01$. $* \star *$ indicated that $\mathrm{P}<0.001$. 
A
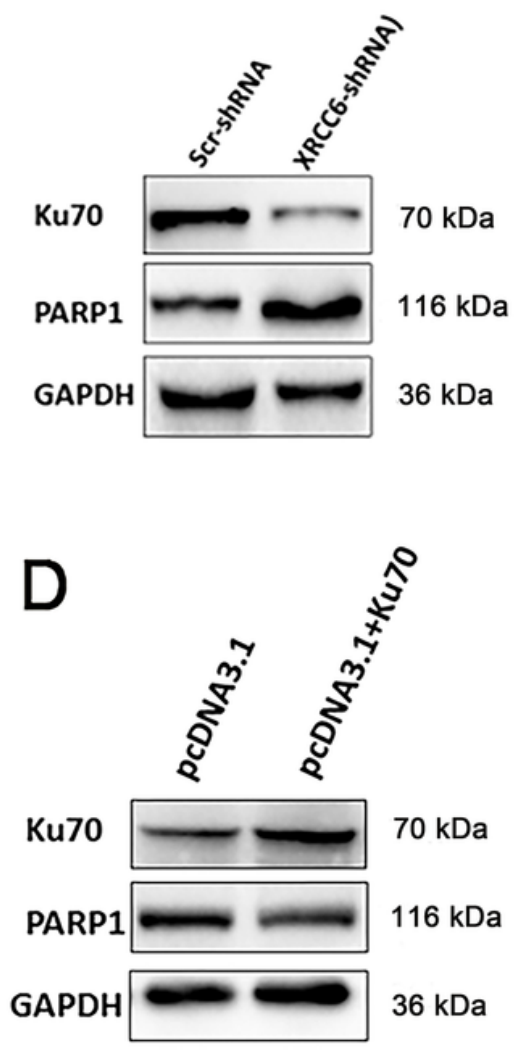

B

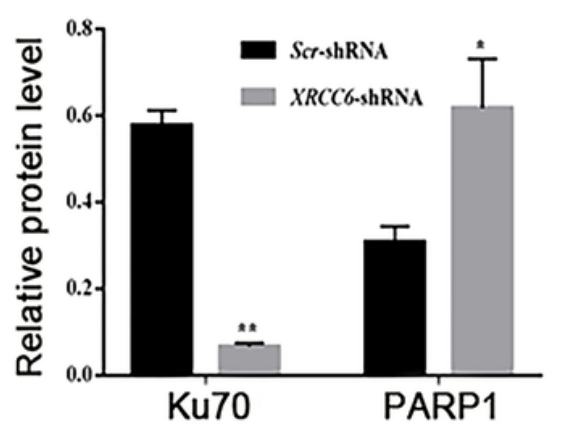

$\mathrm{E}$

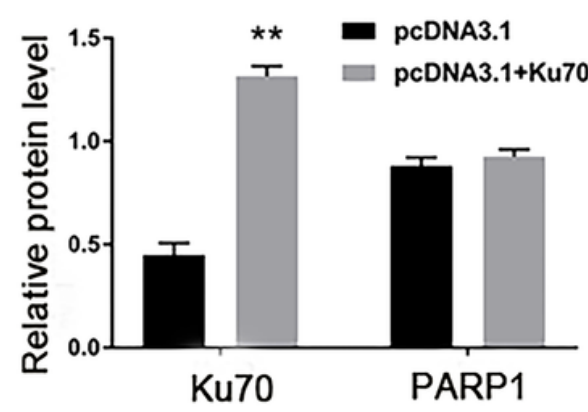

C

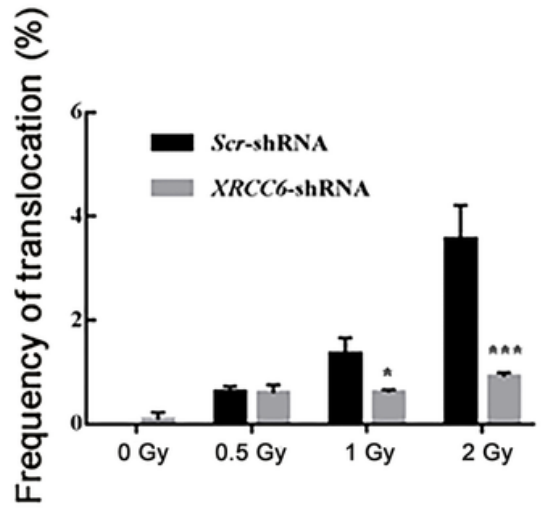

$\mathrm{F}$

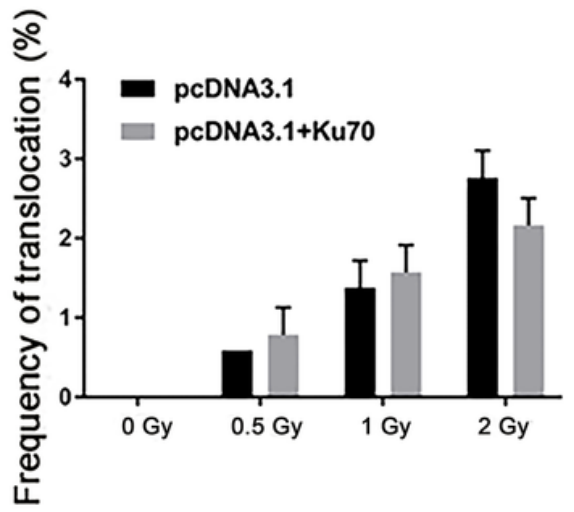

\section{Figure 3}

Ku70 interference decreased the frequency of chromosome translocations. (A) Representative western blots images in Ku70 interference (XRCC6-shRNA) and scr-RNA (control) cells. (B) The protein expression of Ku70 and PARP1 ( $n=3)$ in Ku70 interference (XRCC6-shRNA) and scr-RNA (control) cells. (C) Analysis of the frequency of chromosome translocations $(n=3)$ in Ku70 interference (XRCC6-shRNA) and scr-RNA (control) cells. (D) Representative western blots images in Ku70 overexpression (pcDNA3.1+Ku70) and pcDNA3.1 (control) cells. (E) The protein expression of Ku70 and PARP1 $(n=3)$ in Ku70 overexpression (pcDNA3.1+Ku70) and pcDNA3.1 (control) cells. (F) Analysis of the frequency of chromosome translocations $(n=3)$ in Ku70 overexpression (pcDNA3.1+Ku70) and pcDNA3.1 (control) cells. Sh, short hairpin; Scr, scrambled control. *indicated that $\mathrm{P}<0.05$, **indicated that $\mathrm{P}<0.01$. ***indicated that $\mathrm{P}<$ 0.001 . 
A
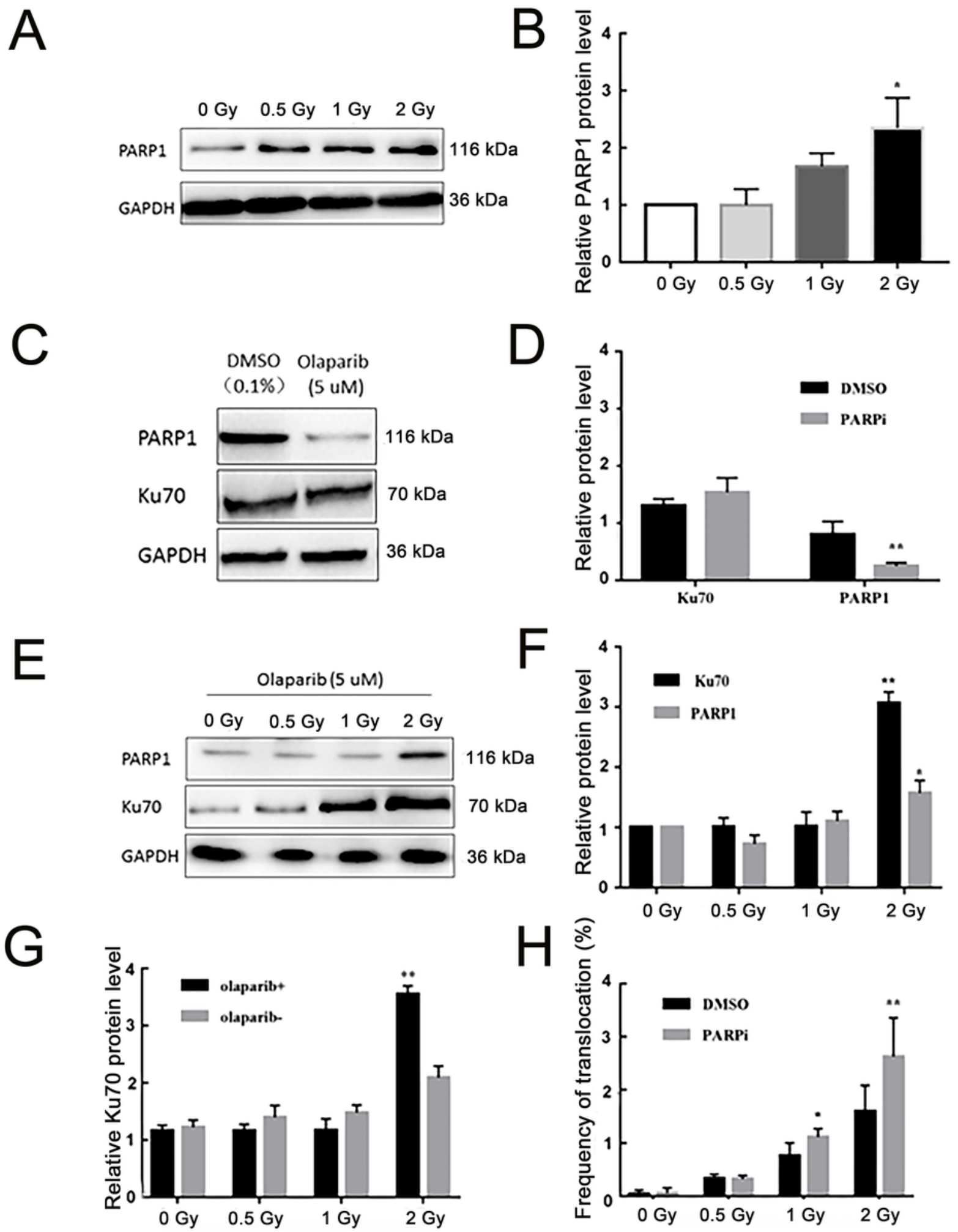

$\mathrm{H}$

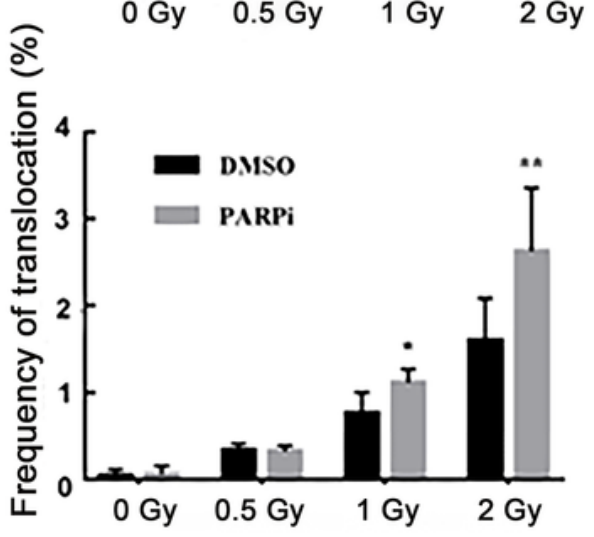

Figure 4

PARP1 inhibitor increased expression of Ku70 and translocations in AHH-1 cell lines. (A) Representative images of the protein expression of PARP1 in cells treated with different dose X-rays. (B) The protein expression analysis $(n=3)$ of PARP1 in cells treated with different dose X-rays. (C) Representative images of the protein expression of PARP1 and Ku70 in cells treated with PARPi (Olaparib). (D) The protein expression analysis $(n=3)$ of PARP1 and Ku70 in cells treated with PARPi (Olaparib). (E) Representative 
images of the protein expression of PARP1 and Ku70 in cells treated with X-rays and in combination with PARPi (Olaparib). ( $F$ ) The protein expression analysis $(n=3)$ of PARP1 and Ku70 in cells treated with $X-$ rays and in combination with PARPi (Olaparib). (G) The protein expression of Ku70 ( $n=3)$ in PBLs treated with X-rays alone (Olaparib-) and in combination with PARPi (Olaparib+). $(\mathrm{H})$ The frequency of chromosome translocations $(n=3)$ in cells treated with X-rays alone (DMSO) and in combination with PARPi (Olaparib), *indicated that $\mathrm{P}<0.05$, **indicated that $\mathrm{P}<0.01$.

A

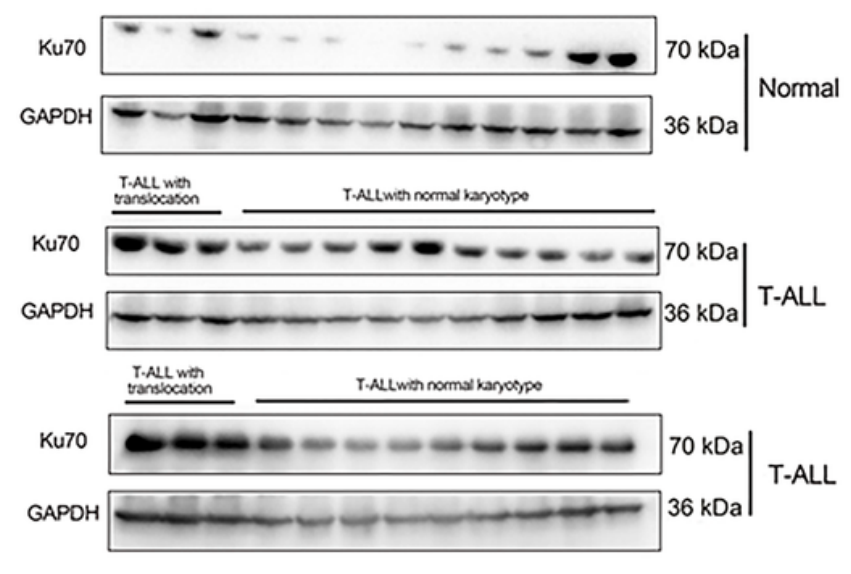

B

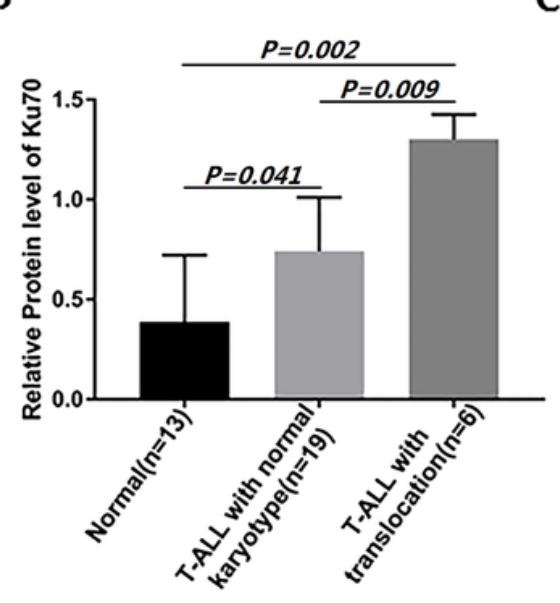

$\mathrm{C}$

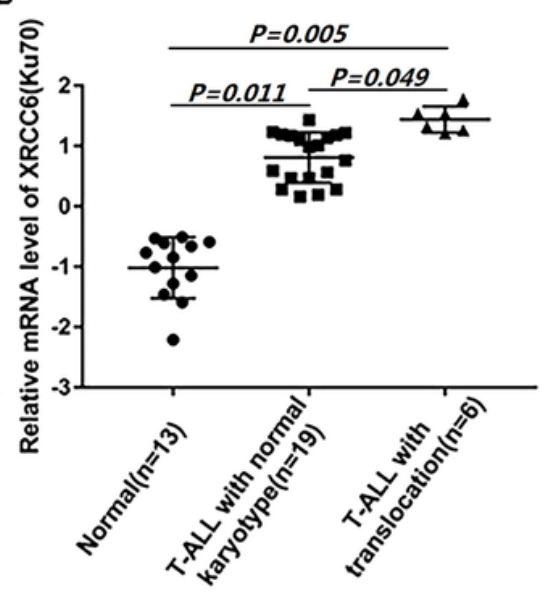

Figure 5

Ku70 protein level in T-ALL was significant higher than that in the control. (A) Representative blots images of Ku70 in T-ALL and normal control. (B) Statistical analysis chart of Ku70 protein levels among groups. The figure shows that Ku70 protein levels are significant high in both T-ALL with translocation and T-ALL with normal karyotype group compared with normal control. (C) XRCC6 mRNA levels in T-ALL samples from Xiangya hospital. X-axis represents different group, Y-axis represents the expression of XRCC6. The scatter plots derived from RT-qPCR comparing expression of XRCC6 gene in normal control (left plots, $n=13)$, T-ALL with normal karyotype (middle plots, $n=19$ ) and T-ALL with translocation (right plots, $n=6$ ). The mRNA levels of XRCC6 are significant higher in T-ALL than in the control. T-ALL: T-cell acute lymphoblastic leukemia.

\section{Supplementary Files}

This is a list of supplementary files associated with this preprint. Click to download.

- Tables1.docx 